Article

\title{
Digital Design of Minimally Invasive Endodontic Access Cavity
}

\author{
Gianluca Gambarini ${ }^{1}$, Massimo Galli ${ }^{1}$, Antonio Morese ${ }^{1}$, Fouad Abduljabbar ${ }^{2}$, \\ Marco Seracchiani ${ }^{1}$, Luigi Vito Stefanelli ${ }^{1}$, Massimo Giovarruscio ${ }^{3}$, Dario Di Nardo ${ }^{1, *}$ (D) and \\ Luca Testarelli ${ }^{1}$ (1)
}

1 Department of Oral and Maxillo-Facial Sciences, Sapienza University of Rome, 00161 Rome, Italy; ggambarini@gmail.com (G.G.); massimo.galli@uniroma1.it (M.G.); moreseantonio@gmail.com (A.M.); marco.seracchiani@uniroma1.it (M.S.); gigistef@libero.it (L.V.S.); luca.testarelli@uniroma1.it (L.T.)

2 Dental Department, King Abdulaziz Medical City, Jeddaah 14611, Saudi Arabia; fgary2001@hotmail.com

3 Department of Therapeutic Dentistry, I.M. Sechenov First Moscow State Medical University, 119991 Moscow, Russia; giovarruscio@gmail.com

* Correspondence: dario.dinardo@uniroma1.it; Tel.: +39-339-3935-527

Received: 3 May 2020; Accepted: 18 May 2020; Published: 19 May 2020

\begin{abstract}
New minimally invasive endodontic cavities have been described and proposed to preserve dentin (and enamel) through strategic access, including point endodontic access cavity (PEAC). There is no consensus to what extent PEAC contributes to tooth's resistance to fracture, because there is no agreement on how PEAC should be performed. The purpose of the present study is to describe and classify four different types of PEACs and to examine if a dynamic navigation system /DNS) could allow planning and precisely executing these cavities in vitro. Forty TrueTooth TM Replica \# 3-001 models, were randomly divided into four identical groups of ten and scanned using a cone bean computed tomography (OP-Maxio 300, Instrumentarium-Kavo, Finland). Then, four different access cavities were planned and performed by using DNS (Navident dynamic navigation system, ClaroNav, Toronto, ON, Canada). For each tooth, a different PEAC was designed to obtain endodontic access to the main mesio-buccal canal (MB1), resulting in a different location of the entry point on the occlusal surface of the tooth. Precision was evaluated by comparing deviation in the inclinations between the planned and real cavity. Data were recorded and statistically analyzed. DNS allowed preparation of minimally invasive "straight line" cavities, with some differences in the accuracy.
\end{abstract}

Keywords: dynamic guide; digital planning; minimally invasive dentistry; conservative endodontic access

\section{Introduction}

In endodontics, the development of safer motor instruments and techniques of instrumentation associated with the support of three-dimensional assessment of dental anatomy is leading clinicians to operate in a more secure environments with enhanced predictability of outcomes: performing minimally invasive access cavities represents the goal of all these improvements [1-4].

Minimally invasive endodontics (MIE) is a clinical approach aiming at performing endodontic techniques and instrumentation, with minimal loss of tooth structure. The basic concept is to disinfect the pulp chamber, then properly clean, shape and fill the root canal systems-without sacrificing extensive occlusal enamel and dentin in the crown and roots. Several studies have shown that the long-term survival of endodontically treated teeth is mostly related to the quality of post-endodontic restorations and to the increased risk of crown and root fractures [5-7].

Many studies evaluating the ability of a tooth to withstand occlusal and functional forces have shown that a tooth with a significant loss of enamel and dentin is distinctly weaker than an intact 
tooth [8-10]. Losing marginal ridges and cusps can significantly and negatively affect tooth strength. Similarly, endodontically treated teeth are usually weaker than untreated ones; hard tissue destruction caused not only by carious lesions, but also excessive, unnecessary aggressive removal of coronal tooth structure during access cavity are considered to be responsible for this loss of strength [11,12].

During the last decades, traditional endodontic cavities (TEC) have been commonly performed without significant changes over time, aiming at allowing a valid access (ideally a straight-line) to the endodontic apex, by reducing coronal interferences [13-15]. More recently, new minimally invasive endodontic cavities have been described and proposed to preserve dentin (and enamel) through strategic access: the conservative endodontic cavities (CEC) and ultraconservative endodontic cavity (UEC) or better known as "ninja access cavity" or PEAC (point endodontic access cavity). These variously sized access cavity designs are both aiming at improving tooth preservation, but they are different in the amount of tissue removal. There is still no accepted classification for these cavities: their differences are based on the assumption that when the diameter of the access preparation is decreased by a half, the operator removes four times less volume of the tooth structure-consequently, a smaller cavity will result in a stronger tooth, with an enhanced resistance to fracture [16]. PEAC is considered the most conservative, minimally invasive approach, but has been criticized because it does not allow to properly clinically detect canal orifices and induces more stressful canal instrumentation [17]. There is no consensus how a PEAC contributes to tooth's strength greatly because there is simply no agreement on how PEAC should be performed. Some authors start from the central fossa, following an oblique projection towards the canal orifices; in other studies a perpendicular straight orifice-directed design is proposed-known as the "truss" access cavity—in which separated cavities are prepared to approach the different canals [18].

The purpose of the present study is to describe and classify four different types of PEACs and to analyze if a dynamic navigation system could allow to planning and precisely executing these cavities in vitro.

\section{Materials and Methods}

A total of 40 artificial resin upper right first molars (TrueTooth ${ }^{\mathrm{TM}}$ Replica \# 3-001 (DELabs, Santa Barbara, CA, USA)) were randomly divided into 4 identical groups $(n=10)$, placed in specific silicon material bases acting as an artificial jaw and scanned by using cone beam computed tomography (CBCT) (OP-Maxio 300, Instrumentarium-Kavo, Finland). The initial sample size $(\mathrm{n}=40)$ was calculated by power analysis ( $p=0.05 \%$ and $80 \%$ interval of confidence).

A unique code was assigned to each group (X1, X2, Y1, Y2). By using dedicated software for implant planning of the Navident dynamic navigation system (DNS) (ClaroNav, Toronto, Ontario, Canada) the DICOM images of dental replicas were acquired and the planning of the access cavity was virtually performed [19]. For each tooth, a different PEAC was designed to get endodontic access to the MB1 canal, with different locations on the occlusal surface of the tooth (Figures 1 and 2)

X1: ultra-conservative access cavity planning, on MB1 canal. Performed on the buccal-palatal plane (buccal view) by planning the opening axis coinciding with the coronal third orifice of the canal (green);

X2: ultra-conservative access cavity planning, on MB1 canal. Performed on the buccal-palatal plane (buccal view) by planning a straight-line access following the axis of the median-apical part of the canal (yellow);

Y1: ultra-conservative access cavity planning, on MB1 canal. Performed on the mesio-distal plane (mesial view) by planning the opening axis coinciding with the coronal third orifice of the canal. (purple);

Y2: ultra-conservative access cavity planning, on MB1 canal. Performed on the mesio-distal plane (mesial view) by planning a straight-line access following the axis of the median-apical part of the canal (blue); 
After the first step (planning), PEACs of each group (Figure 3) were prepared using DNS: the second step was to match the CBCT images with the artificial jaws where replicas were placed, using the Navident "trace registration" system. An optical tracer was glued on the artificial jaw; the teeth surfaces were touched in six different points with a tracer instrument that was also tracked by an optical positioning sensor. During this procedure, the DNS software continuously acquired and recorded points on the traced teeth, and then matched with the CBCT data. A final accuracy check was performed to ensure the precision of such a critical match. Last step was handpiece and burs calibration: this allowed DNS to continuously keep tracing and showing in real time on the computer screen the bur's position. Finally, each planned PEAC was then performed by the same skilled operator, using a precision micro endodontic burr (EndoGuide EG1a-SSWhite) and all canals were located with a size 08 manual instrument (Edge Endo, Albuquerque, New Mexico). Then all 3D replicas were scanned again with CBCT to evaluate and compare if inclination of each PEAC corresponded with the planned one (Figures 4 and 5). A virtual line was traced between the central part of occlusal entry point and the center of the cavity reaching the canal orifice. The angulation of this line was compared to the planned one, which was precisely ending in the central part of the orifice, using a virtual goniometer (Figure 4c). Ideally no differences should be present in terms of angulation; the smaller the difference (in degrees) of the inclinations, the more precise the procedure was. Data were recorded and statistically analyzed using ANOVA and Tukey's post hoc tests (SPSS, Inc., Chicago, IL, USA) and statistical significance was set at $p<0.05$.

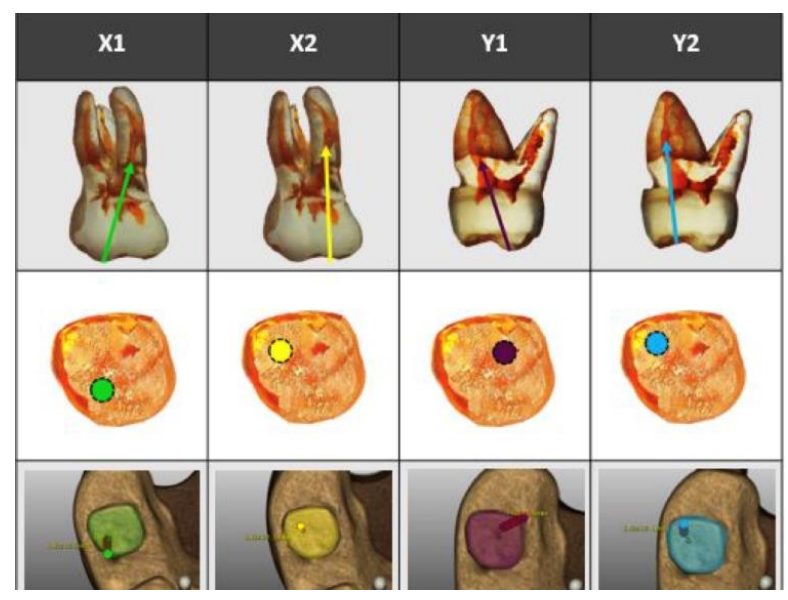

Figure 1. Four different point endodontic access cavities (PEACs) are shown by illustrating for each tooth the planned trajectory, the insertion of the burr on the occlusal surface and 3D rendering of the tooth mounted in the base, with the image of the angulation of the burr for accessing cavity preparation.

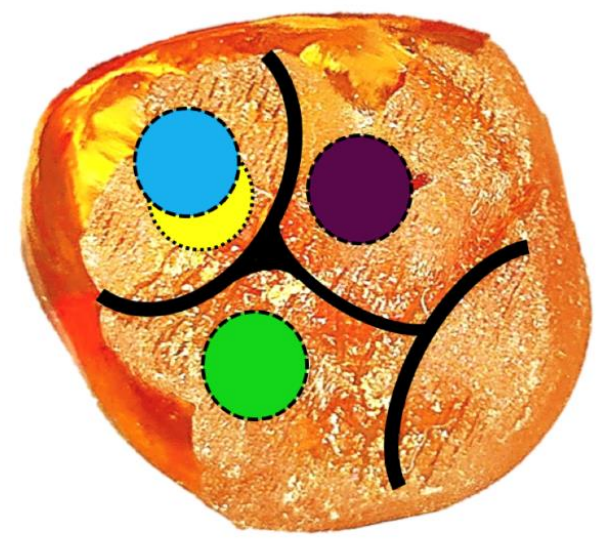

Figure 2. The locations of each different PEAC of Figure 1 are represented on the occlusal surface of the tooth with the same colors. 


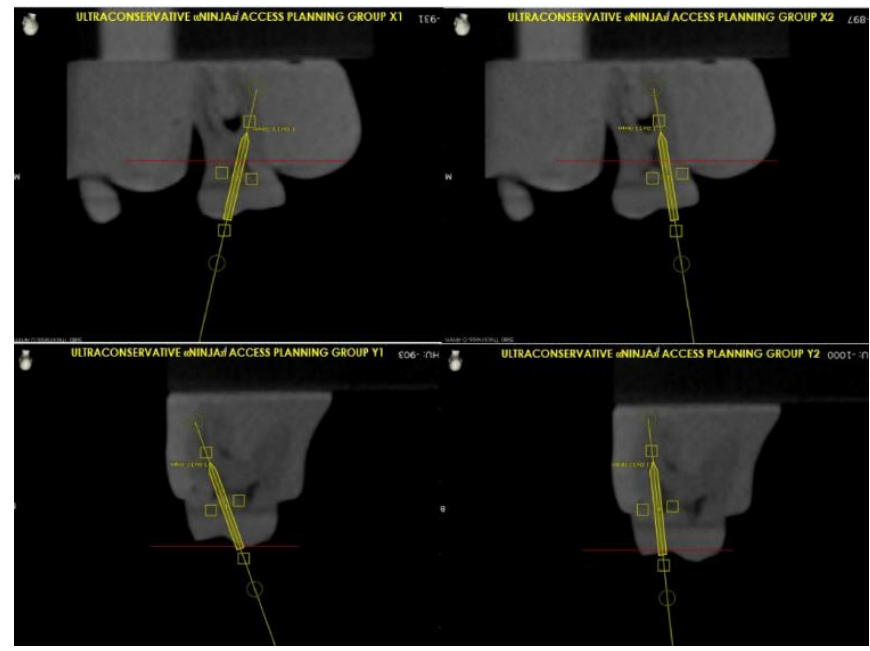

Figure 3. Digital planning of the access cavity using the Navident Software.

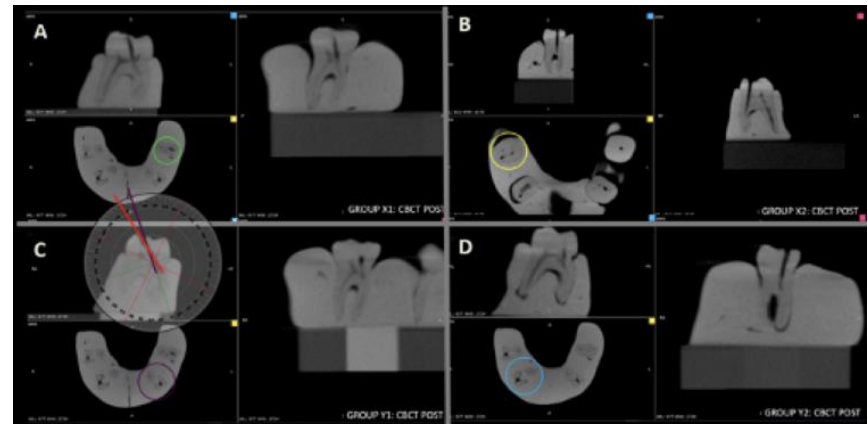

Figure 4. Buccal, mesial and occlusal views of X1 (A), X2 (B), Y1 (C), Y2 (D) group: example cases. In $(\mathbf{C})$ is shown how inclination angle was measured.

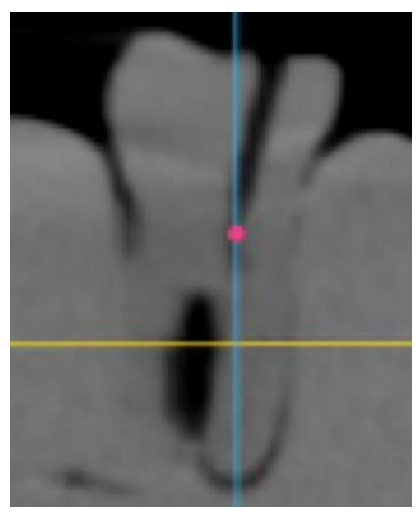

Figure 5. Example of a PEAC, showing a minimally invasive, direct straight-line access from occlusal surface to canal orifice.

\section{Results}

DNS software allowed to plan and perform the different PEACs. Table 1 showed the results evaluation the accuracy of the DNS in performing the planned cavities, by comparing differences in the angulation between planned and real cavities. The $\mathrm{X} 1$ and $\mathrm{Y} 1$ groups showed significantly higher precision than the other two groups.

Among all groups, significant differences were found between the degrees of deviations of the cavities performed hands-free and the ones performed with the DNS $(p<0.05)$. 
Table 1. Deviation from planned view: different superscript letters indicate statistical significance.

\begin{tabular}{cc}
\hline Group & Mean Values and Standard Deviations \\
\hline $\mathrm{X} 1$ & $3.6^{\circ}( \pm 0.4)^{\mathrm{a}}$ \\
\hline $\mathrm{X} 2$ & $3.4^{\circ}( \pm 0.3)^{\mathrm{a}}$ \\
\hline $\mathrm{Y} 1$ & $7.1^{\circ}( \pm 0.8)^{\mathrm{b}}$ \\
\hline $\mathrm{Y} 2$ & $7.2^{\circ}( \pm 0.7)^{\mathrm{b}}$ \\
\hline
\end{tabular}

\section{Discussion}

The initial aim was to identify and classify different PEACs, based on a rational approach: to minimize interferences during endodontic instrumentation by allowing a more direct access and to minimize reduction of teeth's strength, by avoiding unnecessary weakening of tooth structure on the occlusal entry point. Each access cavity was designed taking into consideration the concept of "straight line access" (SLA), aiming at reducing interferences causing instrumentation stress on the rotary instruments. Differently than any other previous study, these SLAs were designed in three dimensions, differing in two parameters. First one is the view since the canals can be viewed both from a bucco-palatal direction and a mesio-distal one. The different views clearly show different original canal trajectories. Consequently, the starting coronal points (SCP) are different in all the cases, being located in different parts of the occlusal surface, to ensure the desired straight-line access. The second aim was to analyze if DNS was able to precisely plan and execute these cavities in vitro. Three-dimensional tooth replicas were selected to ensure that no differences in the anatomy of canals and crowns could alter the comparison between the different PEACs. The results of the present study showed the DNS software, which is clinically used to plan and execute placement of dental implants according to different axis, proved to be very simple to use and provided clear 3D images of treatment planning. The DNS (Table 1) allowed to easily perform PEACs with an overall good accuracy, with statistically significant differences between the cavities; high precision is obviously recommended to avoid unnecessary removal of dentine, but in plastic teeth it is not possible to establish how these variations of the angulation could result in a reduction of the tooth strength. Same limitations, however, could also be present in extracted teeth due to the specific influence of occlusion forces in each patient and the differences in the dimensions of the crowns.

There is no consensus if in mesial canals is better to preserve, partially remove or eliminate any given triangle of dentin, to ensure a more direct straight-line access to the apical third [20,21]. When eliminating these triangles of dentin, canal opening is intentionally relocated slightly away from an external root concavity and toward the greatest bulk of dentin. In a PEAC (Figure 5), this goal can be directly achieved by choosing an end point a few $\mathrm{mm}$ more apically than the orifice, intentionally relocating the end point as previously described; in the classical bucco-palatal view the end point is relocated more mesially, while in the hidden mesio-distal view it is relocated more buccally.

Figure 2 shows the location of the SCPs on the occlusal surfaces: the access cavity $\mathrm{X} 1$ is located on the occlusal face between the MB and the DB cuspids, in an area between the facial sulcus and the central fossa. This type of access cavity could probably lead to a weakening of the marginal ridge, a structure that plays an important role in tooth's strength.

The access cavity $\mathrm{Y} 1$ is located on the occlusal face at the center of the MB cuspid, which is fundamental during chewing; it is a mold for the antagonist element. Theoretically, its involvement and consequent weakening could be a negative factor affecting tooth's strength. Access cavity X2 is located on the occlusal surface, nearby the MB cusp, but away from the marginal ridge. This access cavity can theoretically provide valid instrumentation and avoid involvement of important tooth structure.

The access cavity Y2 is located on the occlusal face, nearby the MB cusp, but more mesial than the $\mathrm{X} 2$ cavity; it can be considered similar to $\mathrm{X} 2$, theoretically allowing a proper access to canal. 
Table 1 showed that DNS allowed a significantly higher accuracy $(p<0.05)$ when compared to $\mathrm{X} 2$ and $\mathrm{Y} 2$, but no significant difference with $\mathrm{Y} 1$. Even if $\mathrm{X} 1$ and $\mathrm{X} 2 \mathrm{PEACs}$ were found to be more accurate, these differences may not be so clinically relevant due to the low recorded angles. On the contrary, the $\mathrm{X} 2$ and $\mathrm{Y} 2$ planning seemed to be almost coincident, and the two groups showed similar results in terms of accuracy; theoretically they could be considered as best options providing both a minimally invasive and an efficient approach. They allowed the preparation of a small "straight line" cavity with a valid access to the MB1 canal, by designing a straight insertion of the rotating instrument. In terms of risk of weakening of tooth structure, $\mathrm{X} 2$ is probably the less invasive PEAC, but these assumptions should be validated by further experimental studies. Overall, DNS was found to be very helpful in following the planned trajectory, with very small deviations recorded: it is not easy to do the same with free-hand instrumentation, especially when the access cavity is angulated. The differences among PEACs in the present study may be related to the fact that endodontists usually visualize and execute access cavity with a bucco-lingual image of the canal trajectory (the one used for $\mathrm{X} 1$ and $\mathrm{Y} 1$ ), while the disto-lingual image of canal trajectory has never been taught and it is commonly not used by clinicians. Therefore, their experience is limited to most common periapical views, which may preclude the visualization of peculiar angulation or position of the coronal orifices.

In the present study artificial 3D replicas were used, instead of extracted teeth. Since the aim was to evaluate cavities with different pathways, the use of identical models allowed to standardize the internal and external anatomy and avoid all possible bias related to unusual anatomy or location of canals, width of the occlusal surfaces and the thickness from occlusal surface to canal orifices. Studies on natural teeth, could give indications on the possibility of get this conservative type of access to all canals in most molar cases.

When comparing these results with those provided by a study on guided implantology [22,23], we found a slight increase in the percentage variations of the angles: this could be a result that both burs and access cavities are much smaller in endodontics. However, $2 \%$ variation in a $1.50 \mathrm{~mm}$ wide implant cavity leads to a $0.03 \mathrm{~mm}$ clinical error, while a $4 \%$ variation in a 0.50 wide endodontic cavity leads to a smaller $0.01 \mathrm{~mm}$ error. In both cases, such variations can be considered very small and allow very precise performance of the planned cavities, both in implantology and endodontics. A future prospect is the creation of customized access cavities for each tooth, by digitally planning the best strategy for each canal, previewing it through a graphic interface, and deciding-depending on the case-which cavity is best, according to above-mentioned concepts of "biologic savings" and "straight line access" [24-26]. The present preliminary study will lead to a series of studies analyzing in vitro, the influence of each cavity in determining instrumentation stress and tooth weakening, if any. The Navident system will not only allow to plan such treatments, but also to execute them precisely and predictably.

\section{Conclusions}

Hence, it is possible to conclude that the digital access cavities design in endodontics offers the possibility of visualizing, before execution, the exact area where the chamber opening should be performed. The DNS technology allowed to perform very precise cavities, with minimal errors associated with any possible inclination of the drill for reaching canal orifices. These excellent experimental results support the clinical use of DNS also in endodontics, as recently suggested by some practitioners, especially when minimally invasive cavities are planned.

Author Contributions: Conceptualization, G.G. and L.T.; methodology, M.G. (Massimo Galli); software, L.V.S.; validation, A.M., M.S. and M.G. (Massimo Giovarruscio); formal analysis, F.A.; investigation, L.V.S.; data curation, G.G.; writing-original draft preparation, G.G.; writing—review and editing, D.D.N.; visualization, D.D.N.; supervision, L.T.; project administration, G.G. All authors have read and agreed to the published version of the manuscript.

Funding: This research received no external funding.

Conflicts of Interest: The authors declare no conflict of interest. 


\section{References}

1. Gambarini, G.; Seracchiani, M.; Piasecki, L.; Valenti Obino, F.; Galli, M.; Di Nardo, D.; Testarelli, L. Measurement of torque generated during intracanal instrumentation in vivo. Int. Endod. J. 2019, 52, 737-745. [CrossRef] [PubMed]

2. Gambarini, G.; Piasecki, L.; Miccoli, G.; Gaimari, G.; Di Giorgio, R.; Di Nardo, D.; Azim, A.A.; Testarelli, L. Classification and cyclic fatigue evaluation of new kinematics for endodontic instruments. Aust. Endod. J. 2019, 45, 154-162. [CrossRef] [PubMed]

3. Gambarini, G.; Miccoli, G.; Seracchiani, M.; Morese, A.; Piasecki, L.; Gaimari, G.; Di Nardo, D.; Testarelli, L. Fatigue Resistance of New and Used Nickel-Titanium Rotary Instruments: A Comparative Study. Clin. Ter. 2018, 169, 96-101.

4. Gambarini, G.; Piasecki, L.; Miccoli, G.; Gaimari, G.; Di Nardo, D.; Testarelli, L. Cone-beam computed tomography in the assessment of periapical lesions in endodontically treated teeth. Eur. J. Dent. 2018, 12, 136-143. [CrossRef] [PubMed]

5. Landys Boren, D.; Jonasson, P.; Kvist, T. Long-term survival of endodontically treated teeth at a public dental specialist clinic. J. Endod. 2015, 41, 176-181. [CrossRef] [PubMed]

6. Fransson, H.; Dawson, V.S.; Frisk, F.; Bjørndal, L.; Kvist, T. Survival of root-filled teeth in the Swedish adult population. J. Endod. 2016, 42, 216-220. [CrossRef] [PubMed]

7. Tang, W.; Wu, Y.; Smales, R.J. Identifying and reducing risks for potential fractures in endodontically treated teeth. J. Endod. 2010, 36, 609-617. [CrossRef]

8. Clark, D.; Khademi, J. Modern molar endodontic access and directed dentin conservation. Dent. Clin. N. Am. 2010, 54, 249-273. [CrossRef]

9. Pereira, J.; McDonald, A.; Petrie, A.; Knowles, J. Effect of cavity design on tooth surface strain. J. Prosthet. Dent. 2013, 110, 369-375. [CrossRef]

10. Boveda, C.; Kishen, A. Contracted endodontic cavities: The foundation for less invasivealternatives in the management of apical periodontitis. Endod. Top. 2015, 33, 169-186. [CrossRef]

11. Panitvisai, P.; Messer, H.H. Cuspal deflection in molars in relation to endodontic and restorative procedures. J. Endod. 1995, 21, 57-61. [CrossRef]

12. Reeh, E.S.; Messer, H.H.; Douglas, W.H. Reduction in tooth stiffness as a result of endodontic and restorative procedures. J. Endod. 1989, 15, 512-516. [CrossRef]

13. Patel, S.; Rhodes, J. A practical guide to endodontic access cavity preparation in molar teeth. Br. Dent. J. 2007, 203, 133-140. [CrossRef] [PubMed]

14. Clark, D.; Khademi, J.A. Case studies in modern molar endodontic access and directed dentin conservation. Dent. Clin. N. Am. 2010, 54, 275-289. [CrossRef]

15. Corsentino, G.; Pedullà, E.; Castelli, L.; Liguori, M.; Spicciarelli, V.; Martignoni, M.; Ferrari, M.; Grandini, S. Influence of Access Cavity Preparation and Remaining Tooth Substance on Fracture Strength of Endodontically Treated Teeth. J. Endod. 2018, 44, 1416-1421. [CrossRef]

16. Plotino, G.; Grande, N.M.; Isufi, A.; Ioppolo, P.; Pedullà, E.; Bedini, R.; Gambarini, G.; Testarelli, L. Fracture Strength of Endodontically Treated Teeth with Differen Access Cavity Designs. J. Endod. 2017, 43, 995-1000. [CrossRef]

17. Saygili, G.; Uysal, B.; Omar, B.; Ertas, E.T.; Ertas, H. Evaluation of relationship between endodontic access cavity types and secondary mesiobuccal canal detection. BMC Oral Health 2018, 18, 121-124. [CrossRef]

18. Abou-Elnaga, M.Y.; Alkhawas, M.A.M.; Kim, H.C.; Refai, A.S. Effect of Truss Access and Artificial Truss Restoration on the Fracture Resistance of Endodontically Treated Mandibular First Molars. J. Endod. 2019, 45, 813-817. [CrossRef]

19. Gambarini, G.; Galli, M.; Stefanelli, L.V.; Di Nardo, D.; Morese, A.; Seracchiani, M.; De Angelis, F.; Di Carlo, S.; Testarelli, L. Endodontic Microsurgery Using Dynamic Navigation System: A Case Report. J. Endod. 2019, 45, 1397-1402. [CrossRef]

20. Moore, B.; Verdelis, K.; Kishen, A.; Dao, T.; Friedman, S. Impacts of contracted endodontic cavities on instrumentation efficacy and biomechanical responses in maxillary molars. J. Endod. 2016, 42, 1779-1783. [CrossRef] 
21. Zogheib, C.; Sfeir, G.; Plotino, G.; Deus, G.; Daou, M.; Khalil, I. Impact of Minimal Root Canal Taper on the Fracture Resistance of Endodontically Treated Bicuspids. J. Int. Soc. Prev. Community Dent. 2018, 8, 179-183. [CrossRef] [PubMed]

22. Buchgreitz, J.; Buchgreitz, M.; Mortensen, D.; Bjørndal, L. Guided access cavity preparation using cone-beam computed tomography and optical surface scans-Anex vivo study. Int. Endod. J. 2016, 49, 790-795. [CrossRef] [PubMed]

23. Stefanelli, L.V.; Mandelaris, G.A.; DeGroot, B.S.; Gambarini, G.; De Angelis, F.; Di Carlo, S. Accuracy of a Novel Trace-Registration Method for Dynamic Navigation Surgery. Int. J. Periodontics Restor. Dent. 2020, 40, 427-435. [CrossRef] [PubMed]

24. Stefanelli, L.V.; DeGroot, B.S.; Lipton, D.I.; Mandelaris, G.A. Accuracy of a Dynamic Dental Implant Navigation System in a Private Practice. Int. J. Oral Maxillofac. Implants 2019, 34, 205-213. [CrossRef]

25. Connert, T.; Zehnder, M.S.; Weiger, R.; Kühl, S.; Krastl, G. Microguided endodontics: Accuracy of aminiaturized technique for apically extended access cavity preparation in anterior teeth. J. Endod. 2017, 43, 787-790. [CrossRef]

26. Connert, T.; Zehnder, M.S.; Amato, M.; Weiger, R.; Kühl, S.; Krastl, G. Microguided Endodontics: A method to achieve minimally invasive access cavity preparation and root canal location in mandibular incisors using a novel computer-guided technique. Int. Endod. J. 2018, 51, 247-255. [CrossRef]

(C) 2020 by the authors. Licensee MDPI, Basel, Switzerland. This article is an open access article distributed under the terms and conditions of the Creative Commons Attribution (CC BY) license (http://creativecommons.org/licenses/by/4.0/). 